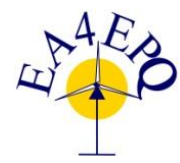

International Conference on Renewable Energies and Power Quality (ICREPQ'13)

Bilbao (Spain), 20 ${ }^{\text {th }}$ to $22^{\text {th }}$ March, 2013

Renewable Energy and Pourer Qualiu. Fournal (RE\&PQJ)

ISSN 2172-038 X, No.11, March 2013

\title{
Cooperative Voltage Control of Distributed Generation and Grid Connected Converter in DC Microgrid
}

\author{
Jong-chan Choi ${ }^{1}$, Ho-young Jeong ${ }^{2}$, Dong-jun Won ${ }^{1}$, Seon-ju Ahn ${ }^{3}$ and Seung-il Moon ${ }^{2}$ \\ ${ }^{1}$ Department of Electrical Engineering \\ Inha University, \\ Incheon, Korea, South \\ Phone/Fax number: +82-32-872-7404, e-mail: whitenpc@gmail.com, djwon@inha.ac.kr \\ ${ }^{2}$ Department of Electrical Engineering \\ Seoul National University, \\ Seoul, Korea, South \\ Phone/Fax number: +82-2-886-3103, e-mail: hyjung@ powerlab.snu.ac.kr, moonsi@ plaza.snu.ac.kr \\ ${ }^{3}$ Department of Electrical Engineering \\ Chonnam University, \\ Gwangju, Korea, South \\ Phone/Fax number: +82-62-530-1738, e-mail: sjahn@ chonnam.ac.kr
}

\begin{abstract}
Many researches on a voltage control method in a DC microgrid has been studied, but almost of them are only focused on control in small scale microgrid such as a building power grid. Whereas it is expected a DC microgrid becomes larger with respect to increasing of a DC energy source and a power load, thus a new control scheme is required for middle or large scale DC microgrids. This paper analyses a voltage drop problem in a radial DC microgrid and proposes a cooperative voltage control scheme with a distributed generation (DG) and a grid connected converter (GCC). The voltage control method with DG and GCC is proposed to minimize an operation cost in DC microgrid and additionally an optimal DG allocation algorithm is proposed for minimization of DG capacity. And finally, the proposed methods are verified by simulation using PSCAD/EMTDC.
\end{abstract}

\section{Key words}

DC microgrid, voltage control method, distributed generation, grid connected converter, optimal DG allocation

\section{Introduction}

In the past several years there has been increasing of interest in DC distribution system which includes distributed generations. A lot of researches study a microgrid with practical experimental systems [1]. Advantages and disadvantages for DC system have been investigated in comparison with those of $\mathrm{AC}$ system. By use of DC, transmittable power can be increased as much as 10 times than those of AC system [2]. Also, DC system can be combined with renewable energy sources not using an inverter. As a result, the efficiency of converted energy can be enhanced from as little as $2.5 \%$ to as much as $10 \%$ than renewable energy sources using an inverter [3].

Many studies have proposed a distribution voltage control in a DC microgrid, and most of them adopted droop concepts to autonomously regulate voltage [4]. In [5], the voltage control method in DC power system that combines fuzzy control and droop control has been presented to manage the energy storage and control voltage. Methods based on droop concepts introduced in these studies provide a stable voltage control, but it is difficult to operate system considering economics.

In this paper, a cooperative voltage control method using grid connected converter (GCC) and a distributed generation (DG) in a radial DC distribution system is proposed to be adapted in larger DC microgrid. Additionally, an optimal allocation algorithm for a DG, a battery energy storage system in this paper, is also proposed because active power of the DG is used for the voltage control in DC microgrid and it is an important issue of economics. Finally, the proposed methodologies are verified by PSCAD/ EMTDC and MATLAB.

\section{Cooperative Voltage Control Scheme in DC Microgrid}

\section{A. Voltage Drop Problem in Radial DC distribution System}

In typical DC microgrid with radial topology, active power flows from the point of common coupling (PCC) to load buses in unidirectional way. Therefore line voltage drop is increased proportionally to load current and line length. A typical voltage profile in DC microgrid is presented as Fig. 1. In this instance, the bus voltages 


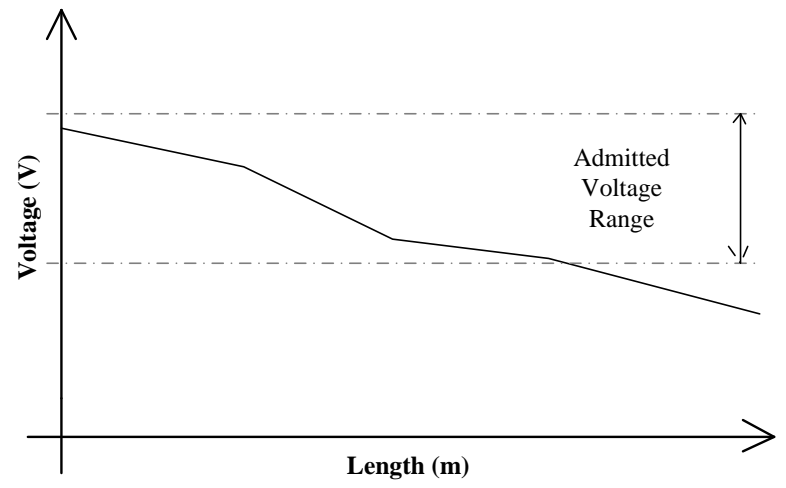

Fig. 1. Voltage Profile in DC Microgrid

would be out of required voltage range as distribution line becomes longer. Moreover, an appliance load would be malfunctioned and voltage stability might be getting worse as the bus voltage is seriously diminished. Therefore voltage control method is required to maintain the voltage within the appropriate range which differs with countries.

In recent researches about a voltage control in small scale DC microgrid, a main topic is focused on how to control a DG and an energy storage device to maintain voltage at certain target bus within required range. However the controlled target bus voltage should be extended to all buses as number of buses is increased as system is expanded. It requires a new control scheme which is different from used methods in small scale DC microgrid, and should guarantee that voltages at all buses are maintained within appropriate range.

\section{B. Cooperative Voltage Control Scheme with Distributed Generation and Grid Connected Converter}

In this paper, a GCC and a controllable DG, which includes energy storage device, are used for a cooperative voltage control in DC microgrid shown as Fig. 2. Advantages of each device could be emphasized through the cooperative control because each device has contrary characteristics.

A GCC acts as an online tap-changing transformer in AC power system from a view point of a voltage control, and it can control bus voltage at the secondary side of the converter. A converter is located at PCC in the proposed control scheme to control voltages at all buses in DC microgrid. Therefore the GCC is used for the cooperative control only, and an additional converter is not considered. However, there might be problem in case some two buses have large voltage difference and only the converter is used for a voltage control. The line voltage drop is increased as bus voltages becomes lower, thus it leads large voltage difference between the highest voltage and the lowest voltage in DC microgrid. And this problem cannot be solved with the GCC only, because the voltage sensitivities of buses with the voltage at secondary side of the GCC are similar to each other.

A DG is one of good solution for the problem mentioned before. A DG can control bus voltage locally in DC distribution system due to the fact that every bus has quite different voltage sensitivity with respect to bus active power. Therefore voltages at all buses can be maintained within required range by voltage control of bus which voltage is the lowest. However, an overall overvoltage or

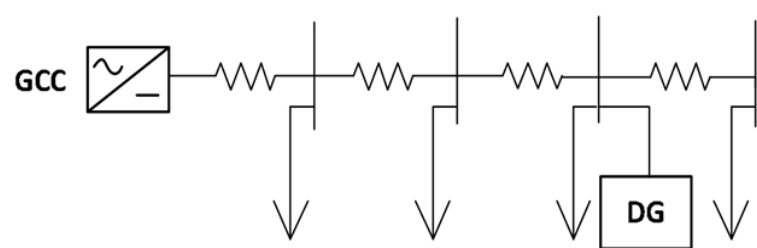

Fig. 2. The Proposed Voltage Control Topology in DC Microgrid

low voltage problems in the entire system cannot be solved if only a DG is used for a voltage control. In addition, generation costs of the DG should be concerned due to the fact that the voltage is controlled by active power in DC microgrid.

Voltage sensitivity with respect to bus active power is closely related to bus voltage. As a bus voltage is high, a bus injection current is quite low when bus active power is injected. It means that the voltage deviation due to bus active power is usually low in this case and voltage sensitivity with respect to bus active power is low. In contrast, if the bus voltage is low, bus injection current due to bus active power is higher than case before. Therefore voltage sensitivity with respect to bus active power becomes higher.

Based on the fact mentioned before, the following cooperative voltage control method is proposed.

- The GCC controls the bus voltage which is the highest in the DC microgrid. The bus that voltage is high has usually lower voltage sensitivity. Therefore large generating power is required if the voltage is controlled by DG, and the GCC is suitable for this case. - The DG regulates the bus voltage which is the lowest in the DC microgrid. The bus that voltage is low has higher voltage sensitivity, and voltage can be regulated effectively with small generating power delivered from the DG.

- The GCC should control voltage as high as possible to reduce line loss. And it leads reduction of power delivered from the DG because the line voltage drop is decreased as the overall voltage is increased.

As a result, the overall overvoltage and the low voltage is controlled by the grid connected converter, and a local voltage problem can be solved by the DG. Therefore, this scheme can reduce an operation cost for voltage control in long term point of view because the power delivered from the DG can be reduced while overall voltage can be regulated.

\section{Algorithm for Optimal DG Allocation}

Voltage control by a DG has large dependency on location of the DG and bus which voltage is controlled. Therefore an optimal DG allocation is essential to control voltage economically with its minimum capacity and power generation from the DG.

In the proposed algorithm, a battery energy storage system which is able to charge and discharge is considered as the DG. Also it is assumed that a generation cost is always constant regardless of system 
Table I. - Definition of Abbreviation in Optimal DG Allocation Algorithm

\begin{tabular}{c|l}
\hline Bus H & Bus with the highest voltage in the DC microgrid \\
\hline Bus L & Bus with the lowest voltage in the DC microgrid \\
\hline Bus I & $\begin{array}{l}\text { Bus with the highest voltage sensitivity affected by } \\
\text { injected active power into Bus L }\end{array}$ \\
\hline $\mathrm{V}_{\text {High }}$ & Voltage at bus which voltage is the highest \\
\hline $\mathrm{V}_{\text {Low }}$ & Voltage at bus which voltage is the lowest \\
\hline $\mathrm{V}_{\text {Max }}$ & Maximum voltage limit in DC microgrid \\
\hline $\mathrm{V}_{\text {Low }}$ & Minimum voltage limit in DC microgrid \\
\hline $\mathrm{N}_{\mathrm{GCC}}$ & $\begin{array}{l}\text { Ratio of voltage at secondary side of GCC on rated } \\
\text { voltage of system }\end{array}$ \\
\hline
\end{tabular}

status. If losses by charging or discharging are small enough to be neglected and the net charging or discharging energy is zero, it can be assumed that generating costs is small enough and it can be ignored. Based on these assumptions, the algorithm is proposed in a way that minimizes the battery capacity to minimize its installation cost. And it will lead minimization of the operation cost because the installation cost is dominant and the generation cost is negligible.

The optimal DG allocation algorithm is presented as follows, and partial derivative of voltage with respect to bus active power is voltage sensitivity which is calculated from inverse of Jacobian matrix. Abbreviation used in algorithm shown as table I.

\section{Step 1.}

- Solve the power flow for the test system and then choose Bus H, Bus L.

- Check voltage violation condition as follows.

$$
\left|V_{\text {High }}-V_{\text {Low }}\right|>V_{\text {Max }}-V_{\text {Min }}
$$

- Check Bus I, and add the Bus I to the list for candidate of optimal DG allocation.

- Battery is not required if (1) is not satisfied.

Step 2.

- Change NGCC in step size until voltage at bus $\mathrm{H}$ exceeds $\mathrm{V}_{\text {max }}$.

- Battery is not required if (1) is not satisfied.

- Check Bus I and add to the list if Bus I is changed.

Step 3.

- Change active power at Bus I as(2). And repeat this procedure until $\Delta$ PI becomes smaller than tolerance value.

$$
\Delta P_{I}=\frac{\left(V_{\text {Max }}-V_{\text {Min }}\right)-\left(V_{\text {High }}-V_{\text {Low }}\right)}{\frac{\partial V_{\text {High }}}{\partial P_{I}}-\frac{\partial V_{\text {Low }}}{\partial P_{I}}}
$$

- If (3) is satisfied, check Bus I and add to the list if Bus I is changed.

\section{Step 4.}

- Repeat step 2 and 3 until voltages at all buses are within the admitted range.

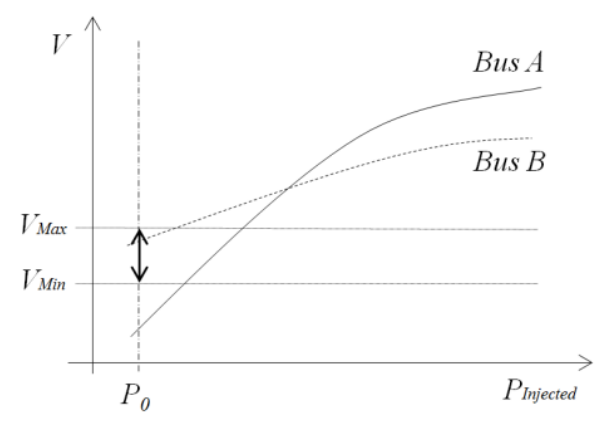

(a) Initial voltage profile

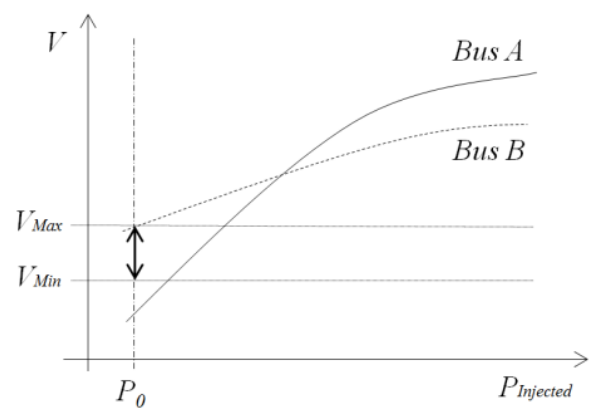

(b) Output voltage of GCC is increased

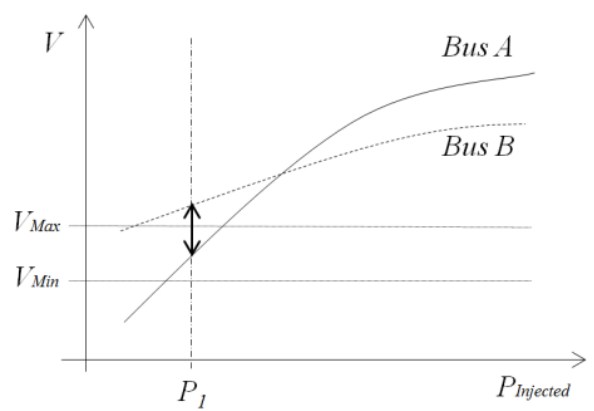

(c) Output power of DG is increased from $P_{0}$ to $P_{1}$

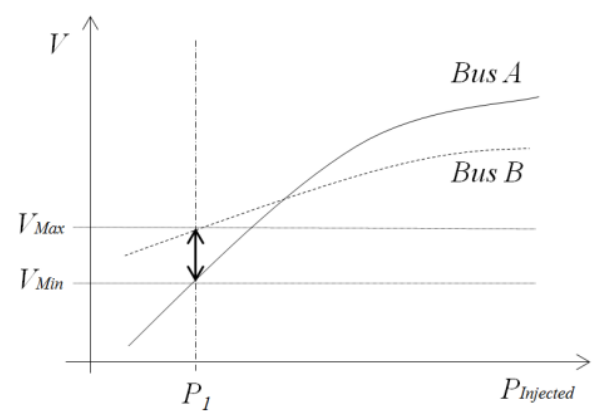

(d) Output voltage of GCC is decreased, and all bus voltages are maintained within admitted range

Fig. 3. Voltage Profile with the Proposed Voltage Control

- In this step, the minimum capacity of battery is determined when the battery storage is located at bus I.

Step 5.

- Repeat through step 1 to step 4 if new buses are added to the list of bus I through step 1 to step 4 .

- Compare the required capacity of the battery storage according to the location of the battery storage. And the bus which required capacity of the battery is the smallest would be the optimal location. 


\section{Cooperative Voltage Control Method}

As mentioned above, a GCC and a DG are used for a voltage control in the proposed method. The GCC would control the bus which voltage is high, and the DG would control the bus which voltage is low. To implement this scheme, voltage at all buses should be obtained by supervisory controller.

The supervisory controller also provides the reference signal to voltage control devices. And it is assumed that location of DG is determined by the proposed allocation algorithm.

To explain that how to control voltage is implemented and effect of control, a simple example is presented. Fig. 3 shows an example of voltage profile when the proposed cooperative voltage control method is adopted.

At initial state, in (a), the voltage at bus $\mathrm{A}$ is out of admitted range and the difference of voltage between bus $\mathrm{A}$ and $\mathrm{B}$ is larger than the difference between $\mathrm{V}_{\max }$ and $\mathrm{V}_{\text {min }}$.

Then the voltage at secondary side of GCC is increased until the voltage at bus $\mathrm{B}$ reaches maximum voltage. Consequently, none of bus voltages exceeds the maximum limit, but the voltage at bus $\mathrm{A}$ is still not within required range.

To increase the voltage at bus A, the output power from DG increases until the voltage difference between bus $A$ and $B$ equals to the difference between $V_{\max }$ and $V_{\text {min }}$. As a result, the voltage difference between two buses is reduced, but the voltage at bus $\mathrm{B}$ exceeds maximum voltage limit due to the output power from DG.

Then the voltage at secondary side of GCC is adjusted until the voltage at bus B becomes smaller than maximum limit. And finally, voltage can be regulated with minimum active power delivered from DG as (d). If voltage at any bus cannot be regulated, repeat this sequence of control until the overall voltages can be maintained within normal range.

\section{Case Studies}

\section{A. Verification of Algorithm for Optimal Positioning Distributed Generation}

The proposed algorithm is verified in the test system shown as Fig. 4. Even though some of the system parameter is not practical, it can be helpful to verify that the proposed algorithm can simulate various situations.

The results of the proposed algorithm are shown as table II and III. In the results, Bus 5, 6 are decided as candidate for optimal location of DG. Among these buses, bus 6 is chosen for optimal location because the required capacity is smaller than bus 5. Thus required capacity of DG for voltage control can be minimized with the proposed

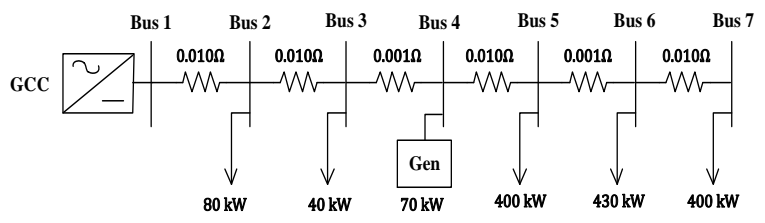

Fig. 4. Test System for Optimal DG Allocation Algorithm allocation algorithm while the overall voltages are maintained within admitted range.

\section{B. Verification of Cooperative Voltage Control Method}

To verify the proposed cooperative voltage control method, a voltage control is implemented in a test system shown as Fig. 5. The test system is connected to AC grid through a GCC and including four load buses and one unit of battery storage. The battery is located at bus 6 by the proposed optimal allocation algorithm. Rating voltage is $311 \mathrm{~V}$, which is peak value of low voltage AC distribution system in South Korea. Loads are constant power load and represented by controllable current source model. Admitted voltage range is 0.95 pu to 1.05 pu. The controllers are designed based on the proposed cooperative voltage control method. Even though maximum and minimum voltage limit is $1.05 \mathrm{pu}$ and 0.95 pu respectively, the bus voltages is regulated within 0.96 to 1.04 pu considering voltage margin.

A effect of the cooperative voltage control method is verified when load power, voltage control and charging modes are changed by following the scenario shown in table IV. And fig. 6 and fig. 7 present the results in accordance with the scenario. Control actions and effects at each time interval are presented as follows.

Table II - Results of Algorithm when DG is Located at Bus 5

\begin{tabular}{c|ccc}
\hline Bus Number & $\begin{array}{c}\text { Generation } \\
\text { Power }(k W)\end{array}$ & $\begin{array}{c}\text { Load } \\
\text { Power }(\mathrm{kW})\end{array}$ & $\begin{array}{c}\text { Bus } \\
\text { Voltage }(\mathrm{pu})\end{array}$ \\
\hline 1 & 7,867 & - & 1.122 \\
2 & - & 80 & 1.050 \\
3 & - & 40 & 0.986 \\
4 & - & -70 & 0.983 \\
5 & 1,445 & 400 & 0.951 \\
6 & - & 430 & 0.950 \\
7 & - & -400 & 0.992 \\
\hline
\end{tabular}

Table III - Results of Algorithm when DG is Located at Bus 6

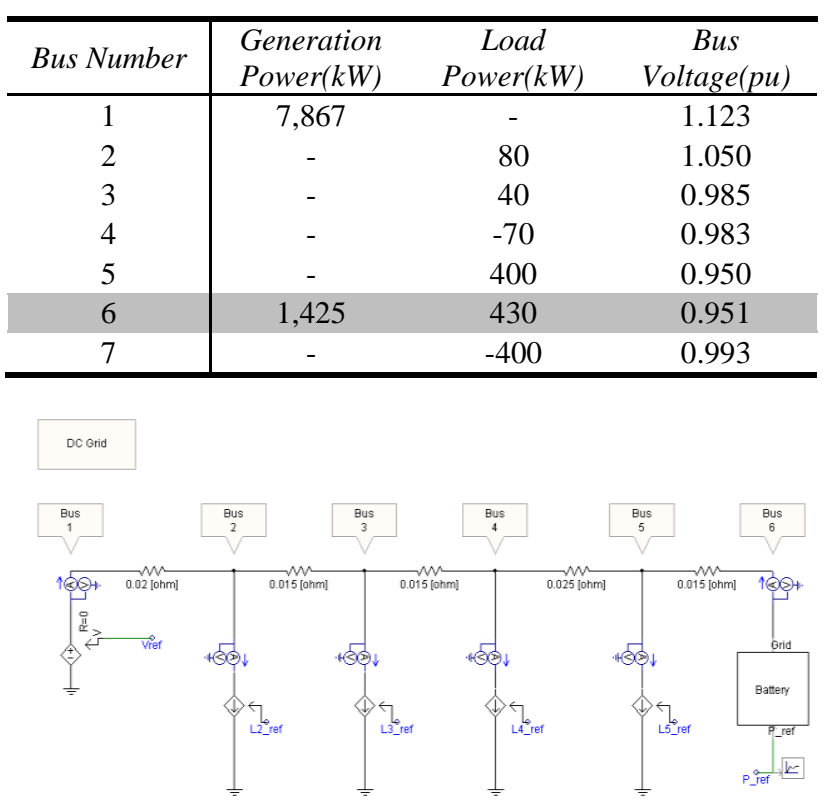

Fig. 5. Test System Implemented in PSCAD/EMTDC 
Table IV - Simulation Scenario

\begin{tabular}{c|c|c|c|c|l|l}
\hline \multirow{2}{*}{ Time } & \multicolumn{4}{|c|}{ Load Power (kW) } & \multicolumn{3}{c}{$\begin{array}{c}\text { Voltage } \\
\text { Control }\end{array}$} \\
\cline { 2 - 7 } & Bus 2 & Bus 3 & Bus 4 & Bus 5 & GCC & Battery \\
\hline $0 \mathrm{~s}$ & \multicolumn{5}{c}{ Initializing } \\
$2 \mathrm{~s}$ & 70 & 50 & 50 & 70 & $\mathrm{X}$ & $\mathrm{X}$ \\
$4 \mathrm{~s}$ & 70 & 50 & 50 & 70 & $\mathrm{O}$ & $\mathrm{X}$ \\
$6 \mathrm{~s}$ & 100 & 70 & 80 & 150 & $\mathrm{O}$ & $\mathrm{X}$ \\
$8 \mathrm{~s}$ & 100 & 70 & 80 & 150 & $\mathrm{O}$ & $\mathrm{O}$ \\
$10 \mathrm{~s}$ & 50 & 70 & 80 & 50 & $\mathrm{O}$ & $\mathrm{X}$ \\
\hline
\end{tabular}

- 0 2 sec: The test system is initializing.

- 2 4 sec: GCC and the battery are not participated in voltage control. The voltage at secondary side of GCC is fixed to $1.0 \mathrm{pu}$ and the delivered power from battery is zero. And bus voltages are not in the required voltage range.

- 4 6 sec: GCC regulates bus voltage which is the highest in DC microgrid. In this case, the voltage at bus 2 is regulated at $1.04 \mathrm{pu}$ by $\mathrm{GCC}$, and all bus voltages are remained in admitted range.

- 6 8 sec: Load consumption powers are increased, and the voltage at bus 5 falls to 0.909 pu. Even though bus voltages are controlled by GCC, some bus voltage is out of admitted range.

- 8 10 sec: GCC and the battery are participated in cooperative voltage control. To boost the voltage which voltage is under minimum limit, active power is delivered from the battery. As a result, all bus voltages are within normal range.

- 10 12 sec: Power consumption of some loads is decreased, therefore power delivered from the battery might be not essential to regulate bus voltages. If load consumptions in DC microgrid are quite small, bus voltages could be regulated even though the battery is charged. Therefore, in this case, charging power is consumed by the battery to recover state of charge of battery.

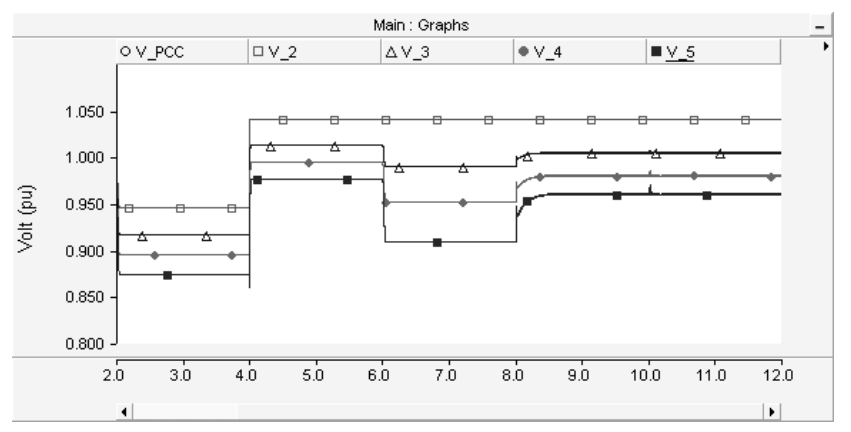

Fig. 6. Result of Simulation Scenario: Bus Voltage

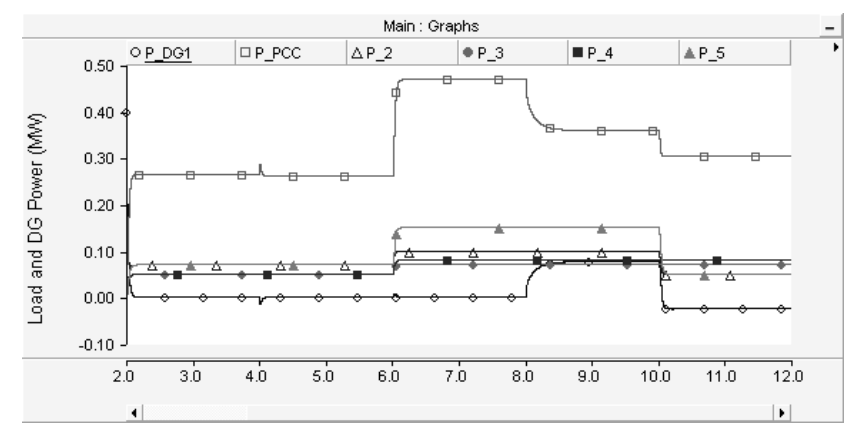

Fig. 7. Result of simulation Scenario: Injected Power

\section{Conclusion}

A voltage control is becoming greatly important issue in a DC microgrid as the DC microgrid becomes larger with high penetration of a renewable energy resource. In addition, active power is required for the voltage control and it means additional cost is imposed for the voltage regulation. Therefore, an economics issue should be considered for a voltage control in a DC microgrid.

In this paper, a cooperative voltage control method using GCC and DG is proposed to be applicable to a medium or large DC microgrid. This method guarantees that all bus voltages are regulated in the admitted range and reduces the delivered power from DG for voltage control. Simulation results show how the proposed method works and how voltage control devices regulate the overall system voltage. Also the required active power of DG for voltage control is closely related to location of DG and it is directly involved in economic issues. Therefore an optimal DG allocation algorithm is proposed to minimize the capacity of DG which is used for a voltage control.

\section{References}

[1] M. Barnes, G. Ventakaramanan, J. Kondoh, R. Lasseter, H. Asano, N. Hataziargyriou, J. Oyarzabal, and T. Green, "Real-World MicroGrids-An Overview," in Proc, IEEE System of Systems Engineering, 2007, pp.1-8.

[2] S.Michael,L.Fangxing,T.Leon.M and O.Burak, "AC vs. DC Distribution: Maximum Transfer Capability," Power and Energy Society General Meeting, Pittsburgh, July 2008.

[3] H.Donald J, "AC Versus DC Distribution Systems - Did We Get it Right?," Power Engineering Society General Meeting, Tampa, June 2007.

[4] Y. Ito, Y. Zhongqing, H Akagi, "DC Microgrid Based Distribution Power Generation System," in Proc, IEEE IPEMC'04, Vol. 3, 2004, pp. 1740-1745.

[5] H. Kakigano, A. Nishino, and T. Ise, "Distribution Voltage Control for DC Microgrid with Fuzzy control and Gainscheduling Control," IEEE Power Electronics and ECCE Asia $8^{\text {th }}$ International Conference. Jeju, 2011. 